\title{
Investigation of Ixodidae ticks infesting camels at slaughterhouse and its potential role in transmitting Coxiella burnetii in Egypt
}

NH Ghoneim

Cairo University Faculty of Veterinary Medicine

Abdel-Moein KA

Cairo University Faculty of Veterinary Medicine

HM Zaher

Cairo University Faculty of Veterinary Medicine

Mai Abuowarda

Cairo University Faculty of Veterinary Medicine

Mai abuowarda ( $\square$ mai.abuowarda@cu.edu.eg )

Cairo University Faculty of Veterinary Medicine https://orcid.org/0000-0003-1631-1666

Research article

Keywords: Imported camels, hard ticks, C. burnetii, Egypt

Posted Date: August 27th, 2020

DOI: https://doi.org/10.21203/rs.2.13281/v2

License: (c) (i) This work is licensed under a Creative Commons Attribution 4.0 International License.

Read Full License 
The authors have withdrawn this preprint from Research Square 